\title{
The Application of Douglas-Peucker Algorithm in Collaborative System for Power Grid Operation Mode Calculation
}

\author{
Chen Jilin ${ }^{1, \mathrm{a}}$, Zhao $\mathrm{Min}^{1}$, Guo Zhonghua ${ }^{1}$, Qiu Weijiang ${ }^{1}$, Chen Yong ${ }^{1}$ and Wang Weixi ${ }^{2}$ \\ ${ }^{1}$ China Electric Power Research Institute, Beijing 100192, China \\ ${ }^{2}$ Institute of Electrical Engineering, Southeast University, Nanjing, China
}

\begin{abstract}
The collaborative system for power grid operation mode calculation implements data management of multi-level paralleling operated dispatching departments indifferent places and joint operation mode calculation, which is based on the calculated data of power grid operation modes. The transient stability analysis monitors the components of electrical variation curve through the network transmission to the web page. When more users and more curves reach a certain extent, the overall simulation time is longer and the curve is stuck because of the limitation of the transmission bandwidth and the refresh rate of the web page. This paper, The Douglas Peucker algorithm is used in system to solve the data transmission of large amount of data delay problem and achieve the transmission of the large amount of data, the overall simulation time of curve in the web page display is decreased, the curves are refreshed smoothly.
\end{abstract}

\section{Introduction}

China's power grid has entered a new period of oversize $\mathrm{AC} / \mathrm{DC}$ hybrid power grid. The operation pressure of large number of power electronic components, such as DC transmission, FACTS and so on, the large-scale access of wind power and photovoltaic power supply is increasing, the electrical connection of the whole network is increasingly close, the characteristics of the power grid are changing deeply, and the coupling relationship between the sections is more complex. Miscellaneous, security and stability levels restrict each other, and the contradiction between resource optimization and safety is prominent. After the AC and DC hybrid power grid is formed, the interaction between UHV and DC transmission system, the coupling of AC and DC system, the mutual restriction between UHV and UHV system are more obvious, which puts forward higher requirements for the lean and integration level of power system analysis and operation control. The continuous development of complex AC and DC power system and its operation control put forward faster and more intelligent requirements for the calculation of power system operation mode and the analysis and processing of the result. with the large-scale construction of power grid access, new energy power generation and construction of interconnected power grid, the grid of size and complexity are increasing, at the same time the power system operation mode calculation of collaborative system has been widely used. The collaborative system for power grid operation mode calculation is based on the calculation data of power grid operation mode, so as to realize the data management and combined mode calculation of multi-level dispatching organization in different places. In the transient stability simulation calculation, the component electrical quantity variation curve is transmitted to the web page through the network. When the user monitors more curves and the number of curves reaches a certain extent, the overall simulation time is longer and the curve is stuck because of the limitation of the transmission bandwidth and the refresh rate of the web page. The operation effect of the system is affected.

Douglas Peucker(DP) algorithm is an iterative adaptive algorithm, the algorithm will reduce the number of points and approximate the curve by a series of points, it has the advantages of translation and rotation invariance, the given threshold and sampling results in DP algorithm. The transient stability simulation can show the changes of the electrical element, but the curve points of the transient simulation are more, and real-time curve transmission is slow, the Douglas Peucker algorithm is applied to the system to solve the data transmission of large amount of data delay problem and achieve the transmission of the large amount of data, the overall simulation time of curve in the web page display is decreased, the curve is refreshed smoothly.

\section{Douglas Peucker algorithm}

The main idea of Douglas Peucker algorithm is the first point and last point of each curve is a straight line, we can get the distance between all the points and line, and find the maximum distance value Dmax, all the points in first and last points are compared with Dmax and tolerance $\mathrm{D}$, if Dmax $<\mathrm{D}$, the middle points of the curve

*Corresponding author: E-Mail: Chenjilin@epri.sgcc.com.cn 
are abandon; if $D \max =\mathrm{D}$, retain the Dmax corresponding to the coordinates of the point, and the curve is divided into two parts, the two parts are used the same method repeat. The diagram analysis is as show in detail:

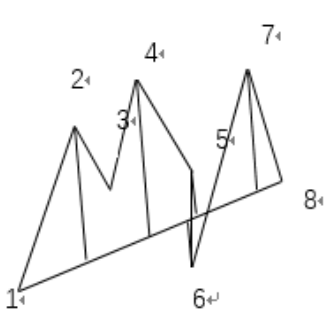

(1)

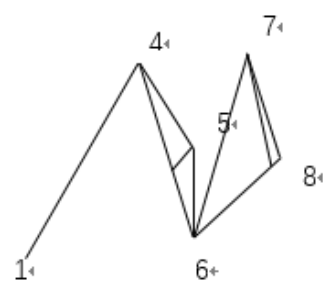

(3)

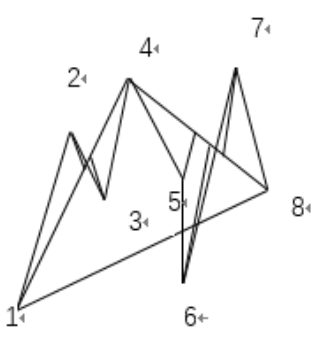

(2)

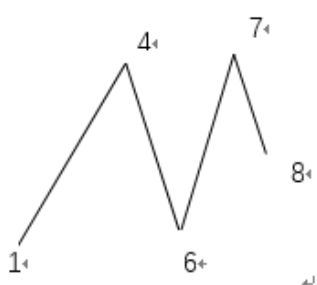

(4)
At the beginning and end of the curve is the straight line, get the distance between the remaining points and the straight line as shown above (1); choose the largest distance which is compared with a threshold, if it is more than the threshold, then retain point which is maximum distance from the straight-line, otherwise they are eliminated between the end of each point in line, as the right figure (2), The fourth point is retained . Keep on this way, the curve is divided into two parts, repeat operation just as first step, second step, the largest distance point is compared to the threshold, the point has been retained and abandoned in turn until there is no point in the end, finally web can get curve point which is the given tolerance difference, as shown in figure (3), (4) The Sixth point and seventh point are retained, all other points are abandoned, and we get the simplification line.

\section{The curve data of power system transient stability analysis}

In the analysis of power system simulation, transient stability analysis curve is a record in the change of each element in the simulation process, the generator power angle and voltage equivalent are shown at the appointed time within the period of time, which reflects the change of state in each time the process of power grid.

In the analysis of power system simulation, the power elements change process of the minimum voltage and the minimum frequency are simulated in a certain period of time, such as all the time is $10 \mathrm{~s}$, the step is 0.01 $\mathrm{s}$, the minimum voltage and minimum frequency of generator bus data are as follows:
Table 1. The data of transient stability simulation.

\begin{tabular}{|c|c|c|c|}
\hline Time & Min voltage & $\begin{array}{c}\text { The number } \\
\text { of bus } \\
\text { Generator }\end{array}$ & Min frequency \\
\hline 0.000 & 0.60193 & 6491 & 1.00000 \\
\hline 0.010 & 0.60194 & 6491 & 0.99998 \\
\hline 0.020 & 0.60173 & 6491 & 0.99994 \\
\hline 0.030 & 0.60152 & 6491 & 0.99989 \\
\hline 0.040 & 0.60146 & 6491 & 0.99982 \\
\hline 0.050 & 0.60144 & 6491 & 0.99974 \\
\hline 0.060 & 0.60143 & 6491 & 0.99965 \\
\hline 0.070 & 0.60145 & 6491 & 0.99955 \\
\hline 0.080 & 0.60146 & 6491 & 0.99944 \\
\hline 0.090 & 0.60148 & 6491 & 0.99933 \\
\hline$\ldots \ldots$ & ......... & $\ldots \ldots$ & $\ldots \ldots$ \\
\hline 9.970 & 0.60180 & 6491 & 0.99804 \\
\hline 9.980 & 0.60182 & 6491 & 0.99804 \\
\hline 9.990 & 0.60185 & 6491 & 0.99807 \\
\hline 10.00 & 0.60187 & 6491 & 0.99812 \\
\hline
\end{tabular}

In power system calculation collaborative system, the transient stability calculation sets a lot of element variables, each variable corresponds to a group of curves, and when much more users submit at the same time, all points must be displayed on the screen through the web transmission, the simulation curves are shown delay and refreshed not smoothly.

\section{The Application of Douglas-Peucker Algorithm In collaborative system}

In collaborative system for power grid operation mode calculation, Multi users monitor curve settings for the transient stability of the power system on the web client, all the setting are submitted to the computing platform. The computing platform accepts the data sent by the client and calls the transient stability calculation, the curve point data according to the user's settings will be output

The curve output data in transmission calculation platform are used in Douglas Peucker algorithm for optimization, After the optimization is completed, the curve point data is written to the JMS server, and the 
user displays the server point data through the web server in real time. At the end of the calculation, the monitoring curve of the user settings is displayed on the web page just as Fig1.

In the power system analysis, the transmission of the curve is the key to ensure the stability and friendliness of the whole system. The number of users of the curve can be set at will. When the monitoring curve reaches tens of thousands, the system should show the curve to the user in the fastest and most stable way.

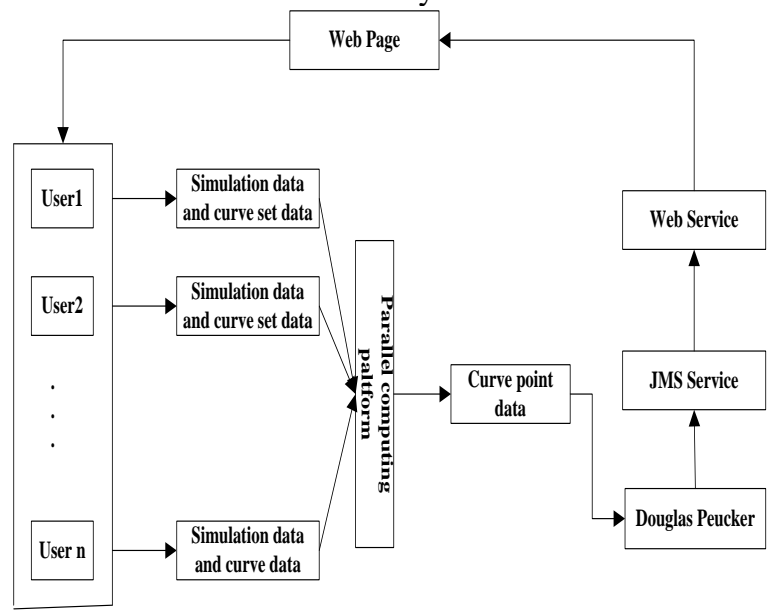

Figure 1. The flow chart of transient stability analysis of collaborative system.

The data processing flow of the curve point of the calculation in platform is shown as figure 2. at First, Determine whether the computation is complete, if not finished, Douglas Peucker algorithm deals the set of segmented data, the specific threshold is 0.01 seconds by default for data optimization, at the end, these datas are written to the JMS server, the system iterates in this way until the calculation is complete.

The determination of the threshold value in the platform depends on the amount of data transmitted or determined by empirical values. the curve is not smooth enough when the threshold is too large; the curve is too fitting when the threshold is too small. Reasonable threshold is also the key factor for the algorithm to be effective.

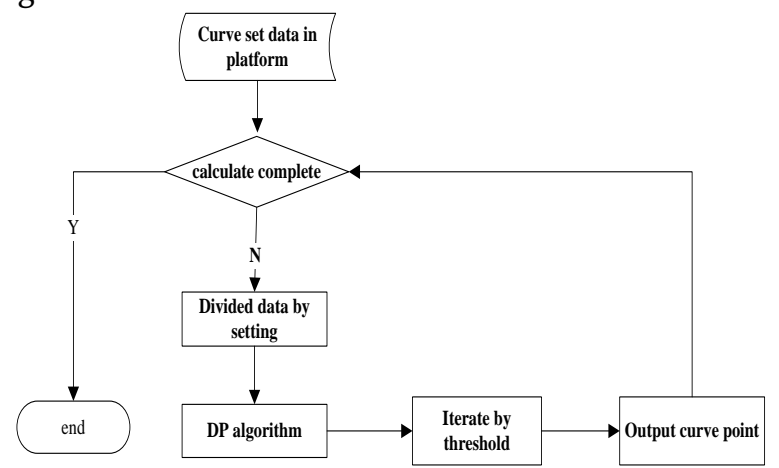

Figure2. The flow chart of Douglas Peucker algorithm used in data processing.

\section{The comparison of the application effect of the algorithm}

The method is applied to the collaborative system for power system operation mode of Henan power grid. The project has been checked and accepted by the provincial level. The transient stability analysis module define the fault set at first, design calculation conditions, the fault set within the scope of monitoring the generator bus voltage setting are fast scanned, after completion of the calculation process, we get the change of components, just as the generator bus voltage curve from 0 to 10 seconds, a total of 1001 points are output according to the total 10 seconds and the step is 0.01 seconds, the curve is shown as in figure 3 :

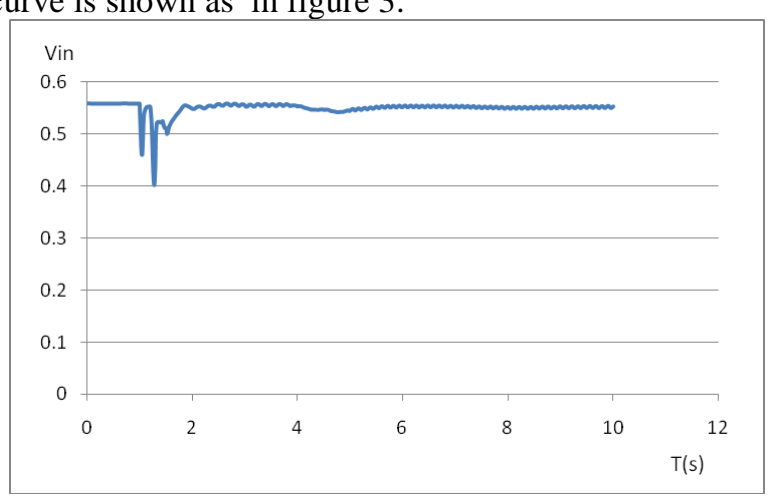

Figure3.The transient stability calculation voltage curve.

Using Douglas Peucker algorithm a series of curve points are optimized and The shape of the curve remains approximately the same, the DP algorithm threshold is 0.01 , the curve point is reduced and the total number is 15 , the curve is shown as in figure 4 :

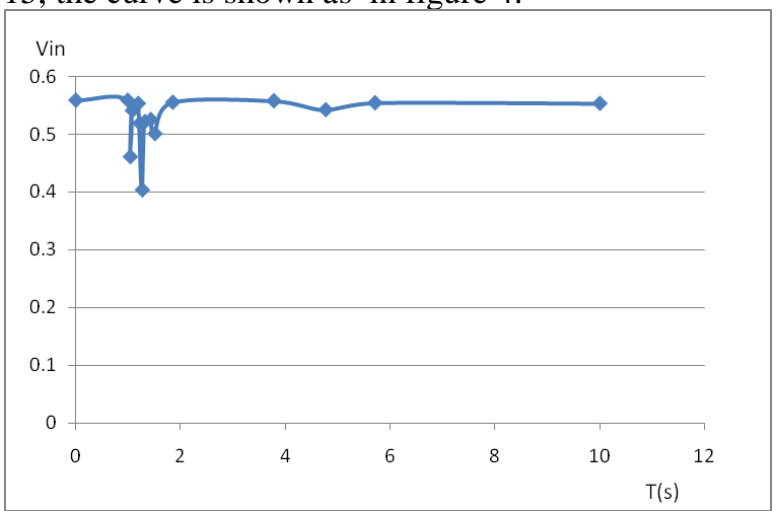

Figure4. The voltage curve optimization by Douglas Peucker algorithm.

With the comparison of figure 3 and figure 4 , it can be seen that the original 1001 points are reduced to 15 points after the Douglas puke algorithm is optimized, and the effect of the curve remains unchanged. After several curve tests, a large number of curves are set up as well. Therefore, in a collaborative computing system, the number of curves can be greatly reduced when the number of curves reaches a certain degree. 


\section{Summary}

The collaborative system for power grid operation mode calculation is based on internet synchronous power grid, offline data, real-time EMS operation data and high performance. The system uses parallel computing technology and distributed computing technology to achieve multi-level scheduling mechanism concurrent data management and joint calculation, the delay problem of large data transmission is solved by using Douglas Peucker algorithm. The system achieves data transmission in the large amount of data and the curve display time is decreased overall simulation on web page and the curve points are refreshed smoothly.

\section{Acknowledgment}

Project Support: Research on Security assessment and decision making technology of large power grid based on spatio-temporal operation data

\section{References}

1. Song Xiaomei, Cheng Changxiu, Zhou Chenghu, et al. Gestalt-based Douglas-Peucker algorithm to keep shape similarity and area consistency of polygons. Sensor Letters, 2013, $11(6 / 7): 1015-1021$

2. He Jin, Fei Lifan . Further study on three dimensional Douglas-Peucker algorithm and its application to generalizationof DEM. Geomatics and Information Science of Wunan University, 2008, 33( 2) : 160-163.

3. Li Bijun, $\mathrm{Fu}$ Hongjun, $\mathrm{Hu}$ Yangyu, et al . Design and implementation of automatic computation and report generation system for annual power grid operation mode. Automation of Electric PowerSystems, 2010, 34(4): 112-115.

4. Zheng Chao, Hou Junxian, Yan Jianfeng, et al. Functional design and implementation of online dynamic security assessment and early warning system . Power System Technology, 2010 , 34(3): 55-60.

5. Mitchel M T, Lei M, Su C J A collaborative control system for mass customization manufacturing . Annals of the CIRP, 1997 , 46(1):): 373-376.

6. Zhang Xingfu, Huang Shaobin . Adaptive neighborhoods based locally linear embedding algorithm . Journal of Harbin Engineering University, 2012, 33( 4) : 489-495

7. Sun Xin, Xia Qing. Power grid operation mode based on elaborating management . Automation of Electric Power Systems 2008，32(21): 14-17
8. State Grid Corporation of China . Smart grid dispatch technology support system design . Beijing : State Grid Corporation of China, 2008.

9. DOUGLAS D H , PEUCKER T K .Algori thms f or the Reduction of the Number of Points Required to Represent a Digitized Line or It s Caricature .The Canadian Cart ographer ,1973 , 10(2):112-122 .

10. Yu Zhuohuang, Lin Hui, Liu Jing. Oriented to massive data power forecast system based on SCADA system[J] . Computer Engineeringand Design, 2010(5): 1114-1117.

11. Zhang Songshu, Chen Yong. Functional Design and Implementation of Collaborative System forPower Grid Operation Mode Calculation. Power System Technology Vol. 36 No. 10

12. Qi G J. Loss-Sensitive Generative Adversarial Networks on Lipschitz Densities[J]. 2017.

13. Mirza M, Osindero S. Conditional Generative Adversarial Nets[J]. Computer Science, 2014:26722680.

14. Yu L, Zhang W, Wang J, et al. SeqGAN: Sequence Generative Adversarial Nets with Policy Gradient[J]. 2016.

15. Silver D, Huang A, Maddison C J, et al. Mastering the game of Go with deep neural networks and tree search[J]. Nature, 2016, 529(7587):484.

16. Lecun $Y$, Bengio $Y$, Hinton G. Deep learning[J]. Nature, 2015,

17. Leverich J, Kozyrakis C. On the energy (in) efficiency of hadoop clusters[J]. ACM SIGOPS Operating Systems Review, 2010, 44(1): 61-65.

18. Kaushik R T, Bhandarkar M. Greenhdfs: towards an energy-conserving, storage-efficient, hybrid hadoop compute cluster[C]//Proceedings of the USENIX annual technical conference. 2010, 109: 34.

19. Goh L K, Veeravalli B, Viswanathan S. Design of fast and efficient energy-aware gradient-based scheduling algorithms heterogeneous embedded multiprocessor systems[J]. IEEE Transactions on Parallel and Distributed Systems, 2009, 20(1): 1-12.

20. Kang J, Ranka S. Dynamic slack allocation algorithms for energy minimization on parallel machines[J]. Journal of Parallel and Distributed Computing, 2010, 70(5): 417-430. 